\title{
Estilos de liderazgo y rendimiento laboral en enfermeros que laboran en el Sector Salud, Juliaca 2019
}

\section{Leadership styles and work performance in nurses working in the Health Sector, Juliaca 2019}

\author{
Luz Yallercco Quispe ${ }^{1}$, Yaned Umire Huarca ${ }^{2}$
}

\begin{abstract}
RESUMEN
Objetivo: determinar la relación entre el estilo de liderazgo y el rendimiento laboral en enfermeros que laboran en el Sector Salud de Juliaca. Metodología: diseño fue no experimental de tipo descriptivo, correlacional y de corte transversal. La muestra estuvo conformada por 120 profesionales de enfermería. Se aplicaron tres instrumentos tipo escala de Likert para la recolección de datos. Resultados: no existe relación significativa entre ambas variables ( $p$ valor 0.555 ), según la prueba Tau-b de Kendall. Así mismo el $74,2 \%$ de los enfermeros tienen un liderazgo transformacional y el $84,2 \%$ evidenciaron rendimiento laboral regular durante el estudio. Conclusión: el estilo de liderazgo no se relaciona con el rendimiento laboral en enfermeros que laboran en el Sector Salud.
\end{abstract}

Palabras clave: liderazgo transformacional, liderazgo transaccional, rendimiento laboral.

\section{ABSTRACT}

Objective: to determine the relationship between leadership style and job performance in nurses who work in the Health Sector of Juliaca. Methodology: design was non-experimental, descriptive, correlational and cross-sectional. The sample was made up of 120 nursing professionals. Three Likert scale instruments were applied for data collection. Results: there is no significant relationship between both variables ( $p$ value 0.555 ), according to the Kendall Tau-b test. Likewise, $74.2 \%$ of the nurses have a transformational leadership and $84.2 \%$ showed regular work performance during the study. Conclusion: leadership style is not related to job performance in nurses working in the Health Sector.

Keywords: transformational leadership, transactional leadership, job performance.

${ }^{1}$ Universidad Peruana Unión, Lima, Perú 


\section{INTRODUCCIÓN}

El liderazgo en enfermería ha cobrado importancia en los últimos años, considerando que es un fenómeno que resulta de una situación determinada (Arce, 1969). Es fundamental para toda estructura organizativa de una institución de Salud. Históricamente los profesionales de enfermería han sobresalido como líderes del equipo de Salud.

Para Castañeda (2010) liderazgo es un conjunto de cualidades y hábitos positivos que motivan a un individuo a dirigir un grupo de personas a metas elevadas, por caminos limitados en principios y valores. Asi mismo Giraldo y Naranjo (2014) afirman que es la influencia que ocurre entre los líderes y sus seguidores, mediante la cual las dos partes pretenden llegar a cambios y resultados reales que reflejen los propósitos que comparten.

El liderazgo transaccional es cuando el líder hace que las cosas se hagan con y a través de los demás (Tracy,2015). Al respecto Fischman (2015) afirma que la jefa de enfermeros trata de satisfacer sus propias necesidades, sólo realiza transacción únicamente cuando existe necesidad siempre esperando algo a cambio. Por otro lado el liderazgo transformacional, centrado en la calidad de atención como un elemento necesario en enfermería, sobre todo cuando el líder abre caminos, motiva, levanta, inspira y traza objetivos que van más allá, no es conformista (Gallardo, 2009). Por ello Felter (2009) afirma que el líder es capaz de crear un cambio, no tiene miedo a los nuevos desafíos, confía en los miembros de su grupo, se preocupa por los demás.

El rendimiento laboral según Altuve y Serrano (2014) es la utilidad o productividad, que las enfermeras aportan, en cuanto al cumplimiento de actividades a las cuales está obligado a ejecutar. Es importante señalar que el bajo rendimiento puede afectar el desempeño del trabajador e incidir en la baja calidad de prestación y ausentismo laboral.

Un estudio realizado en Arequipa donde un $74.51 \%$ de enfermeras practican el liderazgo transaccional, con $19.61 \%$ de enfermeras practican liderazgo transformacional sin embargo el $52.94 \%$ de enfermeras tiene un rendimiento laboral bueno, con $32.03 \%$ presentan rendimiento laboral regular y un rendimiento malo $15.03 \%$. Con la aplicación de la prueba estadística Chi cuadrado, con un nivel de confianza al $99 \%$ y un nivel de error del $1 \%$, se encontró un alto nivel de significancia $(p<0,01)$ entre ambos variables por lo que se acepta la hipótesis que señala que existe relación entre los estilos de liderazgo y satisfacción laboral de las enfermeras del Hospital Región Honorio Delgado.

Dada la importancia del liderazgo para guiar un grupo de personas, se espera que la mayoría desarrolle un liderazgo transformacional ya que puede crear cambios en el profesional enfermero y obtener buenos resultados en cuanto al cuidado humanizado e integral del paciente. Sin embargo es necesario realizar otras investigaciones sobre estilos de liderazgo y rendimiento laboral, tanto a nivel local como a nivel nacional porque existe escasa información.

Por lo tanto, el objetivo del estudio fue determinar la relación entre los estilos de liderazgo y el rendimiento laboral en profesionales de enfermería que laboran en el Sector Salud de Juliaca.

\section{METODOLOGÍA}

Este trabajo de investigación es de enfoque cuantitativo, El diseño del estudio fue no experimental de tipo descriptivo, correlacional y transversal

\section{Participantes}

La muestra estuvo conformada por 120 enfermeras(os), seleccionados por muestreo no probabilístico, distribuidos en 60 enfermeros de la Micro Red Santa Adriana y 60 enfermeros de la Micro Red Cono Sur-Juliaca

\section{Instrumentos}

Los datos se obtuvieron mediante la aplicación de tres cuestionarios: el primero mide el liderazgo transformacional es un cuestionario utilizado y validado en Perú por Rivera (2017) con el objetivo de valorar el grado de liderazgo de las jefas de enfermeras, consta de 39 ítems dividida en 4 dimensiones, tipo escala Likert con una fiabilidad según Alfa de Cronbach de 0.943 y la aplicación de la prueba piloto con 30 profesionales de enfermería. 
El segundo cuestionario mide el liderazgo transaccional, validado en Perú por Collantes (2015), el cuestionario consta de 24 ítems, tipo escala Likert, con una fiabilidad según Alfa de Cronbach de 0.937 , y la prueba de la piloto donde participaron 30 profesionales de enfermería y finalmente, el cuestionario de rendimiento laboral, validado en Perú por Ortega (2016) con el objetivo de medir el rendimiento laboral, con una fiabilidad según Alfa de Cronbach de 0.92 y la prueba piloto de 30 profesionales, el cuestionario también consta de 24 ítems.

\section{Procesamiento de datos}

Se describieron las variables mediante cálculos de frecuencia observada y porcentual para variables nominales u ordinales, para analizar la relación entre los estilos de liderazgo y rendimiento laboral se realizó la prueba estadística Tau-b Kendall que mide variables ordinales vs ordinales y la prueba $\mathrm{T}$ de Student para comparar las medias del puntaje total de la variable.

\section{RESULTADOS}

En la tabla 1 se observa que $74.2 \%$ de las enfermeras(o) practican el liderazgo transformacional, de los cuales 33.3\% laboran en la Micro Red Santa Adriana y $40.8 \%$ laboran en la Micro Red Cono Sur.

Tabla 1

Tipo de liderazgo que ejercen las enfermeras(o) que laboran en la Micro Red Santa Adriana y la Micro Red Cono Sur, Juliaca 2020

\begin{tabular}{|l|c|c|c|c|c|c|c|}
\hline \multicolumn{2}{c|}{ Santa Adriana } & \multicolumn{3}{c|}{ Cono Sur } & \multicolumn{3}{c|}{ Total } \\
\hline \multicolumn{1}{|c|}{ Tipo de liderazgo } & $n$ & $\%$ & $n$ & $\%$ & $n$ & $\%$ \\
\hline Transaccional & 20 & 16.7 & 11 & 9.2 & 31 & 25.8 \\
\hline Transformacional & 40 & 33.3 & 49 & 40.8 & 89 & 74.2 \\
\hline
\end{tabular}

En la tabla 2 se observa que $84.2 \%$ de las enfermeras(o) tienen rendimiento laboral regular, de los cuales $42.5 \%$ laboran en la Micro Red Santa Adriana y $41.7 \%$ de los que ejercen un rendimiento laboral regular laboran en la Micro Red Cono Sur.

Tabla 2

Descripción del rendimiento laboral que ejercen las enfermeras(o) que laboran en la Micro Red Santa Adriana y la Micro Red Cono Sur

\begin{tabular}{|l|c|c|c|c|c|c|c|}
\hline \multicolumn{2}{|c|}{ Santa Adriana } & \multicolumn{3}{c|}{ Cono Sur } & \multicolumn{2}{c}{ Total } \\
\hline \multicolumn{1}{|c|}{ Rendimiento laboral } & $\mathrm{n}$ & $\%$ & $\mathrm{n}$ & $\%$ & $\mathrm{n}$ \\
\hline Malo & 9 & 7.5 & 10 & 8.3 & 19 & 15.8 \\
\hline Regular & 51 & 42.5 & 50 & 41.7 & 101 & 120 \\
\hline
\end{tabular}

En la tabla 3 se observa que no existe relación estadísticamente significativa ( $p$ valor 0.555) entre el estilo de liderazgo y rendimiento laboral, según la prueba Tau de Kendall, aceptando la Ho porque el valor de significancia es mayor a 0.05 .

Tabla 3

Estilo de liderazgo y rendimiento laboral que ejercen los enfermeros en el Sector Salud

\begin{tabular}{lcccc} 
& Valor & Error estándar asintótica & Aprox. Sb & Aprox. Sig \\
$\begin{array}{l}\text { Ordinal por ordinal } \\
\text { Tau-b de Kendall }\end{array}$ & .057 & .096 & .591 & .555 \\
\hline
\end{tabular}




\section{DISCUSIÓN}

Al evaluar el estilo de liderazgo que ejercen las enfermeras(o), se observa que $74,2 \%$ ejercen el liderazgo transformacional y solo $25.8 \%$ el liderazgo transaccional. En un estudio realizado por Lin et al., (2015) demostraron que en el hospital del sector público se practica el liderazgo transformacional (64\%) y liderazgo transaccional (36\%). Resultados diferentes fueron reportados por Rivera (2012) donde demostró que $68 \%$ de las enfermeras (o) del sector salud de primer nivel de atención, ejercían el liderazgo transaccional y solo $32 \%$ el liderazgo transformacional. Al comparar los estilos de liderazgo, se encontró diferencias significativas entre ambas instituciones, en la Micro Red Cono Sur $(40.8 \%)$ y Santa Adriana (33.3\%). Cabe mencionar que ambos resultados tienen similitud en la práctica de liderazgo transformacional. Se refleja un liderazgo transformacional ya que las jefas están en constante capacitación lo que conlleva que las enfermeras de cada Micro Red practiquen el liderazgo transformacional. Donde se practica más el liderazgo transformación en la Micro Red Conos Sur ya que los ítems 2, 3,9 tienen un porcentaje alto de $43.3 \%$ lo cual se obtuvo un alto porcentaje.

El estudio también, evaluó el rendimiento laboral que ejercen las enfermeras, demostrando que $84,2 \%$ tienen rendimiento laboral regular, del mismo modo Peláez y Quispe, (2017) encontraron que $74 \%$ de las enfermeras de la Micro Red Santa Adriana ejercían un tipo de rendimiento laboral intermedio, también Rivera (2002) observo que $70.1 \%$ de las enfermeras del sector salud del Hospital Materno Infantil, presentaron un rendimiento laboral regular. Por lo tanto, en el sector salud de diferentes instituciones presentan un rendimiento laboral intermedio. Al comparar el rendimiento laboral entre ambas instituciones se encontró diferencias mínimas entre el rendimiento laboral entre las enfermeras de la Micro Red Cono Sur $(41.7 \%)$ y Santa Adriana (42.5.0\%). Cabe mencionar que los estudios mencionados muestran resultados muy similares, porque tienen una práctica de rendimiento laboral regular, en ambas instituciones.

Se refleja un rendimiento laboral regular en ambas Micro Redes por sobre carga laboral, y demanda de pacientes. Cada enfermera atiende a 20 pacientes por día en tan solo 12 horas. Seguidamente se quedan horas extras para realizar sus informes del día, por ende, el rendimiento laboral es poco efectiva. Para un buen rendimiento laboral lo ideal es por día se atienda a 10 pacientes ya que correspondería 1 hora por paciente, para brindar un atención de calidad. De tal forma los ítems 24,25 , y 26 tiene un porcentaje de $43.3 \%$ los cuales permitieron alcanzar el puntaje regular, en ambas instituciones.

Se evaluó la relación entre el estilo de liderazgo y rendimiento laboral en la Micro Red Santa Adriana y Cono Sur demostrando que no existe relación estadísticamente significativa ( $P$ valor 0.057). Según Chipa (2017), el análisis de correlación según la prueba estadística de Tau de Kendal demuestra que no existe asociación entre las dos variables, estilos de liderazgo y rendimiento laboral. El análisis de correlación muestra que no hay relación estadísticamente significativa entre estilos de liderazgo y rendimiento laboral, existe la presencia de liderazgo transformacional sin embargo el rendimiento laboral es regular, los resultados muestran rendimiento laboral regular, por la existencia de brechas en relación a los recursos humanos y demanda laboral por los pacientes. El sistema de salud está constituida por Puestos de Salud. En la Micro Red Cono Sur hay 55.123 y .en la Micro Red santa Adriana hay 23785 , decir hay mucha población, y pocos profesionales de salud.

\section{Declaración de financiamiento y de conflictos de interés:}

El estudio fue financiado por los autores, quienes declaran no tener conflictos de interés.

\section{Correspondencia}

Luz Yallercco Quispe

Correo electrónico:

luz.yallerco@upeu.edu.pe

Yaned Umire Huarca

Correo electrónico:

yaned.umire@upeu.edu.pe 


\section{REFERENCIAS BIBLIOGRÁFICAS}

Altuve, E., y Serrano, C. (1999). Relación entre estilos de liderazgo según el modelo de Bolman y Deal y el desempeño laboral. (Tesis de licenciatura, Universidad Andrés Bello)

Arce, A. (1969). Liderazgo, definicion y conceptos (biblioteca conmemorativa ed.). Costa Rica.

Castañeda, L. (2010). Un plan de formacion en liderazgo para jovenes.

Felter, D. (2009). Principio de liderazgo. Brazil: zondervan. Recuperado de: https://books.google. com.pe/books?id=LqbVnQEACAAJ\&dq= principio+de+liderazg o.+brazil:+zondervan. \&hl=es.

Fischman, d. (2015). El lider transformador. san isidro: planeta.

Gallardo, V. (2009). Liderazgo Transformador. Londres. Recuperado de:https://books.google. com.pe/books?id=KVtxAwAAQBAJ\&pg= PT2\&dq=liderazgo+t ransformador.+londres. $\&$ hl=es.
Giraldo, D., y Naranjo, J. (2014). Liderazgo: Desarrollo del concepto, evolución y tendencias. (Tesis de licenciatura, Universidad Del Rosario) 1-42. Recuperado de: http://repository.urosario.edu.co/ bitstream/handle/10336/8672/1026275656 2014. pdf?sequence $=1$

Lin. MacLennan, S., Hunt, N., y Cox, T. (2015). The influences of nursing transformation

Tracy, B. (2015). Liderazgo. Estados Unidos. Recuperado de: https://books.google.com.pe/ books?id=ZUzgCwAAQBAJ\&printsec= frontcover\&dq=liderazgo.+estados+unidos.\&hl=e

Recibido: 09/12/2019

Aceptado: 09/05/2020 